\title{
Correlation analyses between pre- and post-operative adverse events in gastric cancer patients receiving preoperative treatment and gastrectomy
}

Shuang-Xi Li ${ }^{1,2+}$, Sang Hyuk Seo ${ }^{1,3+}$, Yoon Young Choi ${ }^{1}$, Masatoshi Nakagawa ${ }^{1,4}$, Ji Yeong An ${ }^{1,5}$, Hyoung-II Kim', Jae-Ho Cheong ${ }^{1}$, Woo Jin Hyung ${ }^{1}$ and Sung Hoon Noh ${ }^{1 *}$

\begin{abstract}
Backgrounds: Preoperative chemotherapy (PCT) and chemoradiotherapy (PCRT) showed promising results for gastric cancers. However, the influence of preoperative adverse events (AEs) on postoperative complications remains unknown. The aim of this study was to identify correlations between them.

Methods: Clinical data and laboratory findings were retrieved retrospectively for 115 patients who underwent gastrectomy after PCT or PCRT between 2010 and 2013. Preoperative AEs and postoperative complications were classified according to the Common Terminology Criteria for Adverse Events (CTCAE) and Clavien-Dindo (CD) grading systems, respectively. Correlations between CTCAE grades and CD grades were analyzed, and clinical data and laboratory findings were compared among three groups classified according to CD grades: CD0, CD1/2, and CD3/4.

Results: There were 61 (53.0 \%) patients in the CD0 group, 44 (38.3 \%) patients in the CD1/2 group, and 10 (8.7 \%) patients in the CD3/4 group. The CTCAE grades did not correlate with the CD grades. Only estimated blood loss $(P=0.019)$ and transfusion rate $(P<0.001)$ differed among the three $C D$ groups.

Conclusion: There are no correlations between pre- and post-operative adverse events in the terms of severity grades in patients with advanced or metastatic gastric cancer who underwent gastrectomy after PCT or PCRT. Meticulous intraoperative manipulations should be emphasized.
\end{abstract}

Keywords: Gastric cancer, Adverse event, Postoperative complication, Clavien-Dindo, CTCAE

\section{Background}

Gastric cancer is the fifth most prevalent cancer worldwide, and more than $70 \%$ of them occur in less developed countries [1], where they are often diagnosed at an advanced stage. D2 gastrectomy is regarded as the standard surgical treatment for locally advanced gastric cancer (AGC) based on randomized controlled trials [2, 3]. However, further treatment in addition to surgery is required to improve patient survival. The kind of additional

\footnotetext{
* Correspondence: sunghoonn@yuhs.ac

Shuang-Xi Li and Sang Hyuk Seo are joint first authors.

${ }^{\dagger}$ Equal contributors

1 Department of Surgery, Yonsei University Health System, Yonsei University

College of Medicine, 50 Yonsei-ro, Seodaemun-gu, 120-752 Seoul, South Korea

Full list of author information is available at the end of the article
}

treatment varies in different parts of the world: in East Asia, adjuvant chemotherapy after D2 gastrectomy is standard treatment for AGC $[4,5]$, whereas perioperative chemotherapy or postoperative chemoradiotherapy with gastrectomy is standard treatment in the West [6-8].

Recently, the use of preoperative chemotherapy (PCT) has gained increasing interest because of its possible advantages: 1) tumor down-staging increases the possibility of achieving complete surgical resection, 2) early application of chemotherapy may be effective in controlling micrometastases, 3) patients are more tolerable to chemotherapy before surgery, 4) the delivery of 
chemotherapeutic drugs will be enhanced because the blood supply to the tumor is intact, and 5) responses to chemotherapy are easily detected. PCT may even be useful for select patients with metastatic gastric cancer (MGC) prior to surgery $[9,10]$.

Despite these potential advantages, adverse events (AEs) due to PCT or preoperative chemoradiotherapy (PCRT) leading to deterioration of a patient's physical condition could limit the ability to tolerate surgery. This is particularly relevant, as previously reported incidences of severe AEs ranged from 23.8 to $37.6 \%[6,7]$. Thus, there are concerns that PCT or PCRT could increase postoperative morbidity [11]. Although postoperative morbidity after PCT or PCRT has been previously reported to be similar to that noted with surgery alone $[6,7,12]$, to our knowledge, no previous studies have addressed the issue of whether preoperative AEs increase the likelihood or severity of postoperative complications. The aim of this study was to analyze the relationship between preoperative AEs and postoperative complications in patients who underwent gastrectomy for AGC or MGC after receiving PCT or PCRT.

\section{Methods \\ Patients}

Patients were eligible for inclusion in this study if they had histologically-proven primary AGC or MGC, received initial PCT or PCRT, and underwent gastrectomy plus lymphadenectomy at Severance Hospital of Yonsei University between January 2010 and December 2013. The exclusion criteria included the presence of organ dysfunction before initial PCT or PCRT and surgery performed on an emergency basis. No comparative analysis was made to gastric cancer patient undergoing gastrectomy plus lymphadenectomy who did not receive initial PCT or PCRT. The clinical-pathological characteristics and laboratory investigations were retrieved from electronic medical records. The times for which data

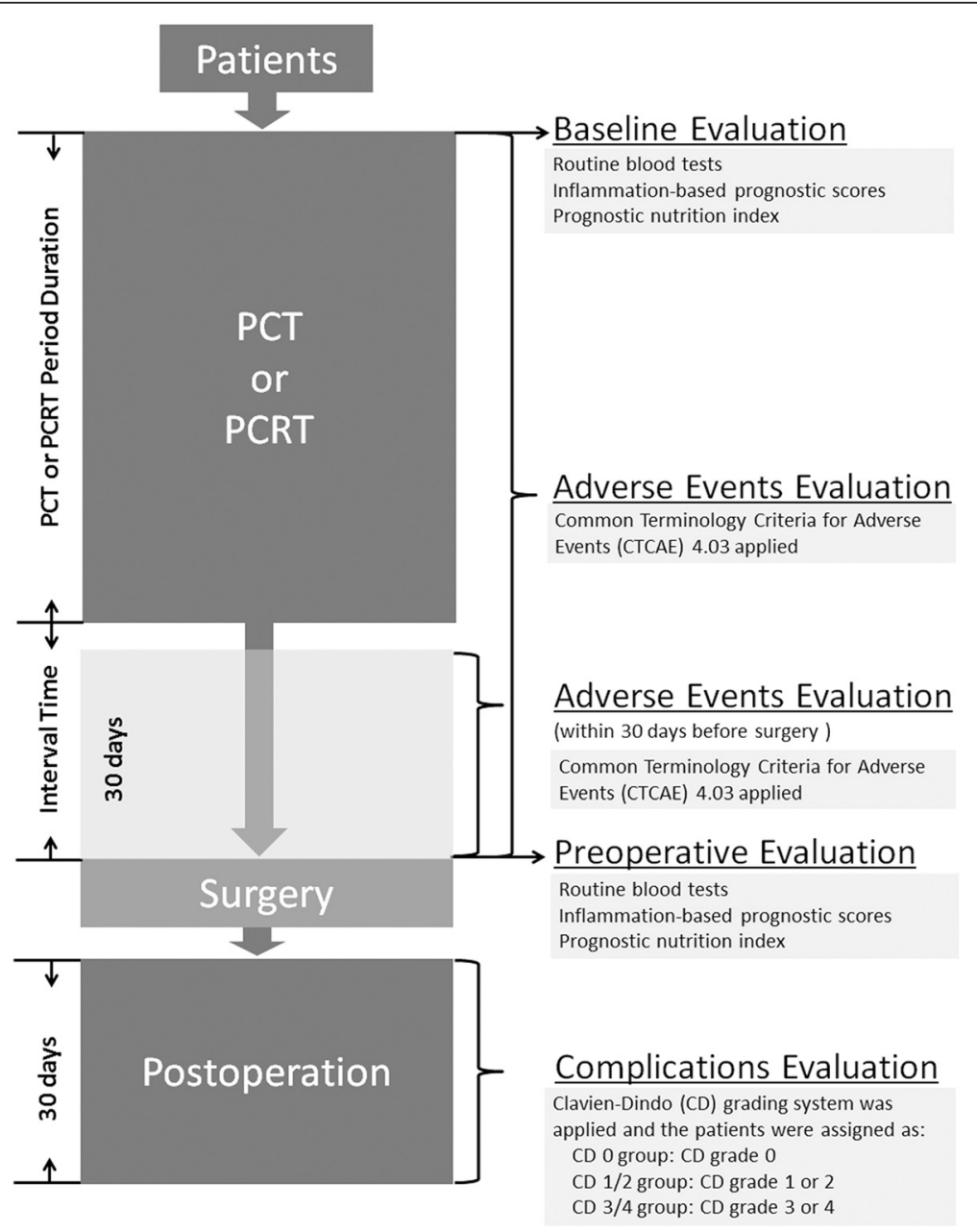

Fig. 1 Study flow diagram. PCT, preoperative chemotherapy; PCRT, preoperative chemoradiotherapy 
were collected are illustrated in Fig. 1. The study was performed in accordance with the Declaration of Helsinki, and was reviewed and approved by the Institutional Review Board of Severance Hospital, Yonsei University College of Medicine (4-2011-0991).

The regimens for PCT or PCRT varied from singleagent to triple-agent, they involved the use of fluorouracil, platinum, taxanes, and/or irinotecan. Intensity modulated radiation was performed using a dose of 40-45 Gy for PCRT. Since all patients received at least one type of fluorouracil-based regimen, we classified the chemotherapy regimens as platinum-containing, taxane-containing, containing both platinum and taxane, and others.

The operations included radical and palliative surgeries. As described in the Japanese Gastric Cancer Treatment Guidelines Version 3.0 [13], radical gastrectomy included resection of at least two-thirds of the stomach and peri-gastric lymphadenectomy with D2 extension. Palliative gastrectomy included resection of the entire gastric lesion or total gastrectomy plus lymphadenectomy with at least D1 extension.

\section{Baseline and preoperative evaluations}

The time point definitions for baseline and preoperative evaluations were the most recent time before the initiation of PCT or PCRT and the surgery (after the final cycle) respectively. The laboratory investigations were collected for evaluations and included 3 categories of routine blood tests:

(1) Complete blood count: hemoglobin, white blood cell, neutrophil, lymphocyte, and platelet.

(2) Hepatorenal function: albumin, alanine transaminase, aspartate transaminase, alkaline phosphatase, and serum creatinine.

(3) Coagulation function: activated partial thromboplastin time, prothrombin, and international normalized ratio.

Additionally, we determined the neutrophil lymphocyte ratio (NLR), platelet lymphocyte ratio (PLR), and prognostic nutritional index (PNI), to evaluate the systemic inflammatory and nutritional status. Preoperatively, C-reactive protein was also recorded, and Glasgow Prognostic Score

Table 1 Clinical characteristics and preoperative treatments ${ }^{a}$

\begin{tabular}{|c|c|c|c|c|c|}
\hline & CD0 group $(n=61)$ & CD1/2 group $(n=44)$ & CD3/4 group $(n=10)$ & Total $(n=115)$ & $P$ Value \\
\hline Age $^{\mathrm{b}}$ (year) & $56.0(27.0-78.0)$ & $56.0(26.0-76.0)$ & $58.5(41.0-75.0)$ & $56.0(26.0-78.0)$ & 0.467 \\
\hline Gender & & & & & 0.452 \\
\hline Male & $36(59.0)$ & $31(70.5)$ & $7(70.0)$ & $74(64.3)$ & \\
\hline Female & $25(41.0)$ & $13(29.5)$ & $3(30.0)$ & $41(35.7)$ & \\
\hline BMI before surgery ${ }^{b}\left(\mathrm{~kg} / \mathrm{m}^{2}\right)$ & $21.9(17.3-30.7)$ & $23.1(17.1-32.4)$ & $21.1(16.9-29.0)$ & $22.3(16.9-32.4)$ & 0.272 \\
\hline Tumor location & & & & & 0.109 \\
\hline Proximal one-third & $12(19.7)$ & $21(47.7)$ & $2(20.0)$ & $35(30.4)$ & \\
\hline Middle one-third & $18(29.5)$ & $8(18.2)$ & $3(30.0)$ & $29(25.2)$ & \\
\hline Distal one-third & $23(37.7)$ & $12(27.3)$ & $4(40.0)$ & $39(33.9)$ & \\
\hline Whole & $8(13.1)$ & $3(6.8)$ & $1(10.0)$ & $12(10.4)$ & \\
\hline Preoperative treatment & & & & & 0.881 \\
\hline PCT & $50(82.0)$ & $34(77.3)$ & $8(80.0)$ & $92(80.0)$ & \\
\hline PCRT & $11(18.0)$ & $10(22.7)$ & $2(20.0)$ & $23(20.0)$ & \\
\hline Cycles $^{b}$ & $5.0(2.0-20.0)$ & $7.0(2.0-19.0)$ & $7.5(2.0-21.0)$ & $5.0(2.0-21.0)$ & 0.082 \\
\hline Duration $^{\mathrm{b}}$ (month) & $3.1(1.2-15.6)$ & $4.5(1.2-18.0)$ & $5.4(1.2-15.2)$ & $3.6(1.2-18.0)$ & 0.061 \\
\hline Interval time ${ }^{\mathrm{b}}$ (month) & $1.1(0.4-5.7)$ & $1.0(0.4-4.5)$ & $1.2(0.5-2.4)$ & $1.1(0.4-5.7)$ & 0.167 \\
\hline Chemotherapy regimen & & & & & 0.922 \\
\hline Platinum-containing & $38(62.3)$ & $25(56.8)$ & $8(80.0)$ & $71(61.7)$ & \\
\hline Taxane-containing & $7(11.5)$ & $6(13.6)$ & $1(10.0)$ & $14(12.2)$ & \\
\hline Containing both platinum and taxane & $14(23.0)$ & $11(25.0)$ & $1(10.0)$ & $26(22.6)$ & \\
\hline Others & $2(3.3)$ & $2(4.5)$ & $0(0.0)$ & $4(3.5)$ & \\
\hline Postoperative hospital stay ${ }^{\mathrm{b}}$ (day) & $8.0(5.0-12.0)$ & $10.0(7.0-27.0)$ & $19.0(11.0-29.0)$ & $9.0(5.0-29.0)$ & $<0.001$ \\
\hline
\end{tabular}

$B M I$ body mass index, $P C R T$ preoperative chemoradiotherapy, $P C T$, preoperative chemotherapy

${ }^{\mathrm{a}}$ Data are presented as number (percentage) unless indicated otherwise

${ }^{\mathrm{b}}$ Data are presented as median (range) 
(GPS) and Prognostic Index (PI) were rated. All these values and factors were calculated and rated as described previously [14].

\section{Preoperative adverse events evaluation}

Only the AEs based on objective laboratory investigations were classified according to the Common Terminology Criteria for Adverse Events (CTCAE) Version 4.03
[15], and they were anemia, leukopenia, neutropenia, febrile neutropenia, lymphocytopenia, thrombocytopenia, increased transaminase, and increased serum creatinine. The grade was determined by the highest CTCAE grade for each event, and the "Total AEs" grade referred to the highest CTCAE grade for all AEs in the study. Furthermore, AEs within 30 days before surgery were evaluated in an attempt to identify correlations between abnormal

Table 2 Surgical and pathological characteristics ${ }^{a}$

\begin{tabular}{|c|c|c|c|c|c|}
\hline & CD0 group $(n=61)$ & CD1/2 group $(n=44)$ & CD3/4 group $(n=10)$ & Total $(n=115)$ & $P$ Value \\
\hline Surgical type & & & & & 0.672 \\
\hline Open & $55(90.2)$ & $42(95.5)$ & $9(90.0)$ & $106(92.2)$ & \\
\hline MIS & $6(9.8)$ & $2(4.5)$ & $1(10.0)$ & $9(7.8)$ & \\
\hline Gastrectomy & & & & & 0.219 \\
\hline Subtotal & $31(50.8)$ & $15(34.1)$ & $5(50.0)$ & $51(44.3)$ & \\
\hline Total & $30(49.2)$ & $29(65.9)$ & $5(50.0)$ & $64(55.7)$ & \\
\hline Surgical radicality & & & & & 0.802 \\
\hline Radical & $37(60.7)$ & $29(65.9)$ & $7(70.0)$ & $73(63.5)$ & \\
\hline Palliative & $24(39.3)$ & $15(34.1)$ & $3(30.0)$ & $42(36.5)$ & \\
\hline Lymphadenectomy & & & & & 0.504 \\
\hline D2 & $47(77.0)$ & $31(70.5)$ & $8(80.0)$ & $86(74.8)$ & \\
\hline D1 plus & $13(21.3)$ & $10(22.7)$ & $1(10.0)$ & $24(20.9)$ & \\
\hline D1 & $1(1.6)$ & $3(6.8)$ & $1(10.0)$ & $5(4.3)$ & \\
\hline Combined resection & & & & & 0.592 \\
\hline Yes & $12(19.7)$ & $7(15.9)$ & $3(30.0)$ & $22(19.1)$ & \\
\hline No & $49(80.3)$ & $37(84.1)$ & $7(70.0)$ & $93(80.9)$ & \\
\hline $\mathrm{EBL}^{\mathrm{b}}(\mathrm{mL})$ & $95(28-300)$ & $126(30-609)$ & $269(50-640)$ & $100(28-640)$ & 0.017 \\
\hline Transfusion & & & & & $<0.001$ \\
\hline Yes & $0(0.0)$ & $17(38.6)$ & $1(10.0)$ & $18(15.7)$ & \\
\hline No & $61(100.0)$ & $27(61.4)$ & $9(90.0)$ & $97(84.3)$ & \\
\hline Borrmann type & & & & & 0.524 \\
\hline I & $6(9.8)$ & $2(4.5)$ & $2(20.0)$ & $10(8.7)$ & \\
\hline ॥ & $12(19.7)$ & $10(22.7)$ & $1(10.0)$ & $23(20.0)$ & \\
\hline III & $32(52.5)$ & $20(45.5)$ & $6(60.0)$ & $58(50.4)$ & \\
\hline IV & 11 (18.0) & $12(27.3)$ & $1(10.0)$ & 24 (20.9) & \\
\hline Lauren type & & & & & 0.569 \\
\hline Intestinal & $30(49.2)$ & $23(52.3)$ & $6(60.0)$ & $59(51.3)$ & \\
\hline Diffuse & $26(42.6)$ & $18(40.9)$ & $2(20.0)$ & $46(40.0)$ & \\
\hline Mixed & $5(8.2)$ & $3(6.8)$ & $2(20.0)$ & $10(8.7)$ & \\
\hline TNM Stage & & & & & 0.846 \\
\hline yр 0 & $4(6.6)$ & $1(2.3)$ & $1(10.0)$ & $6(5.2)$ & \\
\hline yp la-lb & $14(23.0)$ & $7(15.9)$ & $1(10.0)$ & $22(19.1)$ & \\
\hline yp Ila-Ilb & $6(9.8)$ & $6(13.6)$ & $2(20.0)$ & $14(12.2)$ & \\
\hline yp IIla-IIIc & 15 (24.6) & $15(34.1)$ & $3(30.0)$ & 33 (28.7) & \\
\hline yp IV & $22(36.1)$ & $15(34.1)$ & $3(30.0)$ & $40(34.8)$ & \\
\hline
\end{tabular}

EBL, Estimated blood loss; MIS, Minimal invasive surgery

${ }^{a}$ Data are presented as number (percentage) unless indicated otherwise

${ }^{\mathrm{b}}$ Data are presented as median (range) 
physical conditions in the month prior to surgery and postoperative complications.

\section{Postoperative complications evaluation}

Postoperative complications within 30 days after surgery were classified according to the Clavien-Dindo (CD) grading system [16]. In our study, pulmonary-related complications included atelectasis, pleural effusion, or pneumonia, and infection-related complications consisted of gastrointestinal (GI) tract leakage, abdominal abscess, wound infection, pneumonia, or pyelonephritis. Patients with a temperature $>38.5^{\circ}$ centigrade and in whom other complications were subsequently excluded were diagnosed with an "unexplained fever", which was classified as a grade 1 complication. The patients were assigned to one of the following three groups based on the severity of postoperative complications: no complications (CD0 group); grade 1 or 2

Table 3 Incidence of AEs during PCT or PCRT according to CTCAE and Clavien-Dindo grades ${ }^{\mathrm{a}}$

\begin{tabular}{|c|c|c|c|c|c|}
\hline & CD0 group $(n=61)$ & CD1/2 group $(n=44)$ & CD3/4 group $(n=10)$ & Total $(n=115)$ & $P$ Value \\
\hline \multicolumn{5}{|l|}{ Anemia } & \multirow[t]{4}{*}{0.099} \\
\hline Grade 0 & $6(5.2)$ & $6(5.2)$ & $0(0.0)$ & $12(10.4)$ & \\
\hline Grade 1/2 & $48(41.7)$ & $27(23.5)$ & $6(5.2)$ & $81(70.4)$ & \\
\hline Grade 3/4 & $7(6.1)$ & $11(9.6)$ & $4(3.5)$ & $22(19.1)$ & \\
\hline \multicolumn{5}{|l|}{ Leukopenia } & \multirow[t]{4}{*}{0.326} \\
\hline Grade 0 & $24(20.9)$ & $9(7.8)$ & $4(3.5)$ & $37(32.2)$ & \\
\hline Grade 1/2 & $21(18.3)$ & $21(18.3)$ & $3(2.6)$ & $45(39.1)$ & \\
\hline Grade 3/4 & $16(13.9)$ & $14(12.2)$ & $3(2.6)$ & $33(28.7)$ & \\
\hline \multicolumn{5}{|l|}{ Neutropenia } & \multirow[t]{4}{*}{0.244} \\
\hline Grade 0 & $16(13.9)$ & $4(3.5)$ & $3(2.6)$ & $23(20.0)$ & \\
\hline Grade 1/2 & $19(16.5)$ & $16(13.9)$ & $3(2.6)$ & $38(33.0)$ & \\
\hline Grade 3/4 & $26(22.6)$ & $24(20.9)$ & $4(3.5)$ & $54(47.0)$ & \\
\hline \multicolumn{5}{|c|}{ Febrile neutropenia } & \multirow[t]{3}{*}{0.829} \\
\hline Grade 0 & 49 (42.6) & $35(30.4)$ & $9(7.8)$ & $93(80.9)$ & \\
\hline Grade 3/4 & $12(10.4)$ & $9(7.8)$ & $1(0.9)$ & $22(19.1)$ & \\
\hline \multicolumn{5}{|c|}{ Lymphocytopenia } & \multirow[t]{4}{*}{0.748} \\
\hline Grade 0 & $10(8.7)$ & $9(7.8)$ & $2(1.7)$ & $21(18.3)$ & \\
\hline Grade 1/2 & $41(35.7)$ & $29(25.2)$ & $5(4.3)$ & 75 (65.2) & \\
\hline Grade 3/4 & $10(8.7)$ & $6(5.2)$ & $3(2.6)$ & $19(16.5)$ & \\
\hline \multicolumn{5}{|c|}{ Thrombocytopenia } & \multirow[t]{4}{*}{0.798} \\
\hline Grade 0 & 39 (33.9) & $26(22.6)$ & $8(7.0)$ & $73(63.5)$ & \\
\hline Grade 1/2 & $19(16.5)$ & $16(13.9)$ & $2(1.7)$ & $37(32.2)$ & \\
\hline Grade 3/4 & $3(2.6)$ & $2(1.7)$ & $0(0.0)$ & $5(4.3)$ & \\
\hline \multicolumn{5}{|c|}{ Increased ALT/AST } & \multirow[t]{3}{*}{0.718} \\
\hline Grade 0 & 34 (29.6) & $28(24.3)$ & $6(5.2)$ & $68(59.1)$ & \\
\hline Grade 1/2 & $27(23.5)$ & $16(13.9)$ & $4(3.5)$ & 47 (40.9) & \\
\hline \multicolumn{5}{|l|}{ Increased Scr } & \multirow[t]{3}{*}{1.000} \\
\hline Grade 0 & $58(50.4)$ & $41(35.7)$ & $9(7.8)$ & $108(93.9)$ & \\
\hline Grade 1/2 & $3(2.6)$ & $3(2.6)$ & $1(0.9)$ & $7(6.1)$ & \\
\hline \multicolumn{5}{|l|}{ Total AEs } & \multirow[t]{4}{*}{0.138} \\
\hline Grade 0 & $0(0.0)$ & $1(0.9)$ & $0(0.0)$ & $1(0.9)$ & \\
\hline Grade 1/2 & $29(25.2)$ & $11(9.6)$ & $3(2.6)$ & $43(37.4)$ & \\
\hline Grade 3/4 & $32(27.8)$ & $32(27.8)$ & $7(6.1)$ & $71(61.7)$ & \\
\hline
\end{tabular}

AEs adverse events, ALT alanine transaminase, $A S T$ aspartate transaminase, CTCAE common terminology criteria for adverse events, $P C R T$ preoperative chemoradiotherapy, $P C T$ preoperative chemotherapy, $\mathrm{Scr}$ serum creatinine

${ }^{\text {a }}$ Data are presented as number (incidence) 
complications (CD1/2 group); and grade 3 or 4 complications (CD3/4 group).

\section{Statistical analyses}

Nominal data are presented as number (percentage), scale data are presented as median (range), and AEs and complications are presented as number (incidence). Differences in nominal data and AEs among groups were detected by Chi-square tests or Fisher's exact tests and differences in scale data among groups were detected by Kruskal-Wallis tests. Correlation analyses between $\mathrm{CD}$ grades and ordinal data, including CTCAE grades, GPS, and PI, were performed by Spearman's correlation tests. A $P$ value less than 0.05 (two-sided) was considered statistically significant. All analyses were performed with SPSS $^{\circ}$ version 22.0 (SPSS Inc., Chicago, IL, USA).

\section{Results}

\section{Clinical-pathological characteristics}

A total of 115 patients were included in this study. There were 74 males (64.3\%) and 41 females (35.7\%).
The majority of patients received PCT, whereas only 23 patients $(20.0 \%)$ received PCRT. The postoperative stay differed among the three groups $(P<0.001)$. PCT or PCRT consisted of a median of $5.0(1.0-21.0)$ cycles, and there were no significant differences among groups regarding other treatment-related parameters (Table 1). The operations primarily involved open surgery, total gastrectomy, radical resection, and D2 lymphadenectomy. Only estimated blood loss $(\mathrm{EBL})(P=0.017)$ and intra/postoperative transfusion rate $(P<0.001)$ were significantly different among groups (Table 2 ).

\section{Incidence and type of preoperative adverse events}

Overall, the incidence rates for AEs during PCT or PCRT were as follows (in descending order): anemia $(89.6 \%, 103 / 115)$, lymphocytopenia $(81.7 \%, 94 / 115)$, neutropenia (80\%, 92/115), leukopenia $(67.8 \%, 78 / 115)$, increased transaminase $(40.9 \%, 47 / 115)$, thrombocytopenia $(32.2 \%, 37 / 115)$, febrile neutropenia $(19.1 \%, 22 / 115)$, and increased serum creatinine $(6.1 \%, 7 / 115)$. The total AEs incidence was $99.1 \%(114 / 115)$. For serious AEs classified as CTCAE grade $3 / 4$, the incidence rates were as follows

Table 4 Incidence of AEs within 30 days before surgery according to CTCAE and Clavien-Dindo grades ${ }^{\mathrm{a}}$

\begin{tabular}{|c|c|c|c|c|c|}
\hline & CD0 group $(n=61)$ & CD1/2 group $(n=44)$ & CD3/4 group $(n=10)$ & Total $(n=115)$ & $P$ Value \\
\hline Anemia & & & & & 0.724 \\
\hline Grade 0 & $23(20.0)$ & $13(11.3)$ & $2(1.7)$ & $38(33.0)$ & \\
\hline Grade 1/2 & $36(31.3)$ & $30(26.1)$ & $8(7.0)$ & $74(64.3)$ & \\
\hline Grade 3/4 & $2(1.7)$ & $1(0.9)$ & $0(0.0)$ & $3(2.6)$ & \\
\hline Leukopenia & & & & & 0.196 \\
\hline Grade 0 & $38(33.0)$ & $27(23.5)$ & $10(8.7)$ & $75(65.2)$ & \\
\hline Grade 1/2 & $19(16.5)$ & $15(13.0)$ & $0(0.0)$ & 34 (29.6) & \\
\hline Grade 3/4 & $4(3.5)$ & $2(1.7)$ & $0(0.0)$ & $6(5.2)$ & \\
\hline Neutropenia & & & & & 0.740 \\
\hline Grade 0 & $38(33.0)$ & $26(22.6)$ & $8(7.0)$ & $72(62.6)$ & \\
\hline Grade 1/2 & $16(13.9)$ & $12(10.4)$ & $2(1.7)$ & $30(26.1)$ & \\
\hline Grade 3/4 & $7(6.1)$ & $6(5.2)$ & $0(0.0)$ & $13(11.3)$ & \\
\hline Lymphocytopenia & & & & & 0.720 \\
\hline Grade 0 & $31(27.0)$ & $19(16.5)$ & $6(5.2)$ & $56(48.7)$ & \\
\hline Grade 1/2 & $27(23.5)$ & $24(20.9)$ & $4(3.5)$ & $55(47.8)$ & \\
\hline Grade 3/4 & $3(2.6)$ & $1(0.9)$ & $0(0.0)$ & $4(3.5)$ & \\
\hline Thrombocytopenia & & & & & 0.577 \\
\hline Grade 0 & $45(39.1)$ & 34 (29.6) & $9(7.8)$ & $88(76.5)$ & \\
\hline Grade 1/2 & $16(13.9)$ & $10(8.7)$ & $1(0.9)$ & $27(23.5)$ & \\
\hline Total AEs & & & & & 0.421 \\
\hline Grade 0 & $14(12.2)$ & $8(7.0)$ & $1(0.9)$ & $23(20.0)$ & \\
\hline Grade 1/2 & $36(31.3)$ & $28(24.3)$ & $9(7.8)$ & $73(63.5)$ & \\
\hline Grade 3/4 & $11(9.6)$ & $8(7.0)$ & $0(0.0)$ & 19 (16.5) & \\
\hline
\end{tabular}

AEs adverse events, CTCAE common terminology criteria for adverse events 
(in descending order): neutropenia (47.0\%, 54/115), leukopenia (28.7\%,33/115), anemia (19.1\%, 22/115), lymphocytopenia $(16.5,19 / 115)$, febrile neutropenia $(12.2 \%, 14 / 115)$, and thrombocytopenia $(4.3 \%, 5 / 115)$. The total serious AEs incidence was $61.7 \%$ (71/115). The distributions of $\mathrm{AE}$ severity (ratios of grade 0 , grade $1 / 2$, and grade $3 / 4$ ) were comparable among groups (Table 3 ).

For AEs within 30 days before surgery, the AE incidence rates were as follows (in descending order): anemia (67.0\%, 77/115), lymphocytopenia (51.3\%, 59/115), neutropenia (37.4 \%, 43/115), leukopenia (34.8\%, 40/115), and thrombocytopenia $(23.5 \%, 27 / 115)$. The total AEs incidence was $80.0 \%(92 / 115)$, including a $16.5 \%(19 / 115)$ incidence of grade 3/4 AEs. The distributions of AE severity (ratios of grade 0 , grade $1 / 2$, and grade $3 / 4$ ) did not differ among groups (Table 4).

\section{Incidence and type of postoperative complications}

There was no postoperative mortality, and the morbidity rate was $47.0 \%(54 / 115)$. The most frequent complication was pulmonary-related complications, the most common minor complications (CD1/2) were pulmonary-related complications and transfusion, and the most common serious complication (CD3/4) was GI tract leakage/abscess (Table 5).

\section{Correlation analyses of preoperative adverse events and postoperative complications}

Correlation analyses were performed between the CTCAE grade of AEs during PCT or PRCT and the CD grade of postoperative complications using Spearman's correlation tests. The results revealed no correlations between the two grading systems (Fig. 2). Similarly, no correlations were noted when comparing the CTCAE grades of AEs within 30 days before operation and the CD grade of postoperative complications (Fig. 3).

\section{Differences in laboratory tests, inflammation-based prognostic scores, and nutrition index}

During baseline and preoperative evaluations, all laboratory test results were comparable among groups, with no statistically significant differences. Similarly, the NLR, PLR, and PNI at baseline and preoperatively failed to exhibit significant differences among groups. According to Spearman's correlation tests, neither the preoperative GPS nor PI was correlated with the postoperative complications CD grade (Table 6).

\section{Discussion}

In this retrospective study, we focused on the relationship between preoperative $\mathrm{AEs}$ and postoperative complications in patients with AGC or MGC who received PCT or PCRT. The incidence of both total AEs and total
Table 5 Postoperative complications ${ }^{a}$

\begin{tabular}{|c|c|c|c|}
\hline & $\begin{array}{l}\text { CD1/2 group } \\
(n=44)\end{array}$ & $\begin{array}{l}\text { CD3/4 group } \\
(n=10)\end{array}$ & $\begin{array}{l}\text { Total }^{\mathrm{b}} \\
(n=115)\end{array}$ \\
\hline \multicolumn{4}{|l|}{ Non-surgery-related } \\
\hline Pulmonary-related & $17(14.8)$ & $3(2.6)$ & $20(17.4)$ \\
\hline Deep vein thrombus & $1(0.9)$ & $0(0.0)$ & $1(0.9)$ \\
\hline Unexplained fever & $7(6.1)$ & $0(0.0)$ & $7(6.1)$ \\
\hline Transfusion & $17(14.8)$ & $1(0.9)$ & $18(15.7)$ \\
\hline Others $^{c}$ & $3(2.6)$ & $1(0.9)$ & $4(3.5)$ \\
\hline \multicolumn{4}{|l|}{ Surgery-related } \\
\hline Wound discharge/infection & $5(4.3)$ & $2(1.7)$ & $7(6.1)$ \\
\hline Intestinal obstruction & $3(2.6)$ & $1(0.9)$ & $4(3.5)$ \\
\hline Fluid collection & $9(7.8)$ & $1(0.9)$ & $10(8.7)$ \\
\hline $\begin{array}{l}\text { Gastrointestinal tract leakage/ } \\
\text { abscess }\end{array}$ & $1(0.9)$ & $6(5.2)$ & $7(6.1)$ \\
\hline \multicolumn{4}{|l|}{ Complication type } \\
\hline Not infection-related & $26(22.6)$ & $3(2.6)$ & $29(25.2)$ \\
\hline Infection-related & $12(10.4)$ & $7(6.1)$ & $19(16.5)$ \\
\hline \multicolumn{4}{|l|}{ Clavien-Dindo grade } \\
\hline Grade 1 & $15(13.0)$ & NA & $15(13.0)$ \\
\hline Grade 2 & $29(25.2)$ & NA & $29(25.2)$ \\
\hline Grade 3 & NA & $8(7.0)$ & $8(7.0)$ \\
\hline Grade 4 & NA & $2(1.7)$ & $2(1.7)$ \\
\hline
\end{tabular}

NA not applicable

${ }^{\mathrm{a}}$ Data are presented as number (incidence)

${ }^{\mathrm{b}}$ Included 61 patients in CD0 group

Included pyelonephritis, hydronephrosis, re-admission for nutrition support, and renal dysfunction

serious AEs were extremely high compared to previous studies $[6,7,17]$. The 5.0 median cycles of PCT or PCRT in our study may have contributed to this discrepancy, as it is reasonable to expect a higher incidence of AEs in our population who received extensive preoperative treatment. Even in the time period very close to surgery (within 30 days before surgery), we observed a relatively high incidence of total AEs. The AEs associated with PCT or PCRT would definitely negatively affect a patient's physical condition, which may subsequently influence the postoperative course; thus, concerns have arisen about the safety of performing gastrectomy and lymphadenectomy for such vulnerable patients.

A correlation between poor physical status and postoperative morbidity was previously reported by Jung et al. [18], who indicated that patients with preoperative anemia had a higher morbidity rate after radical gastrectomy than patients without. By contrast, the CTCAE grades for AEs in our study did not correlate with postoperative CD grades. A similar result was reported by Reim et al. [19], who noted that preoperative leukopenia due to neoadjuvant chemotherapy had no significant effect 


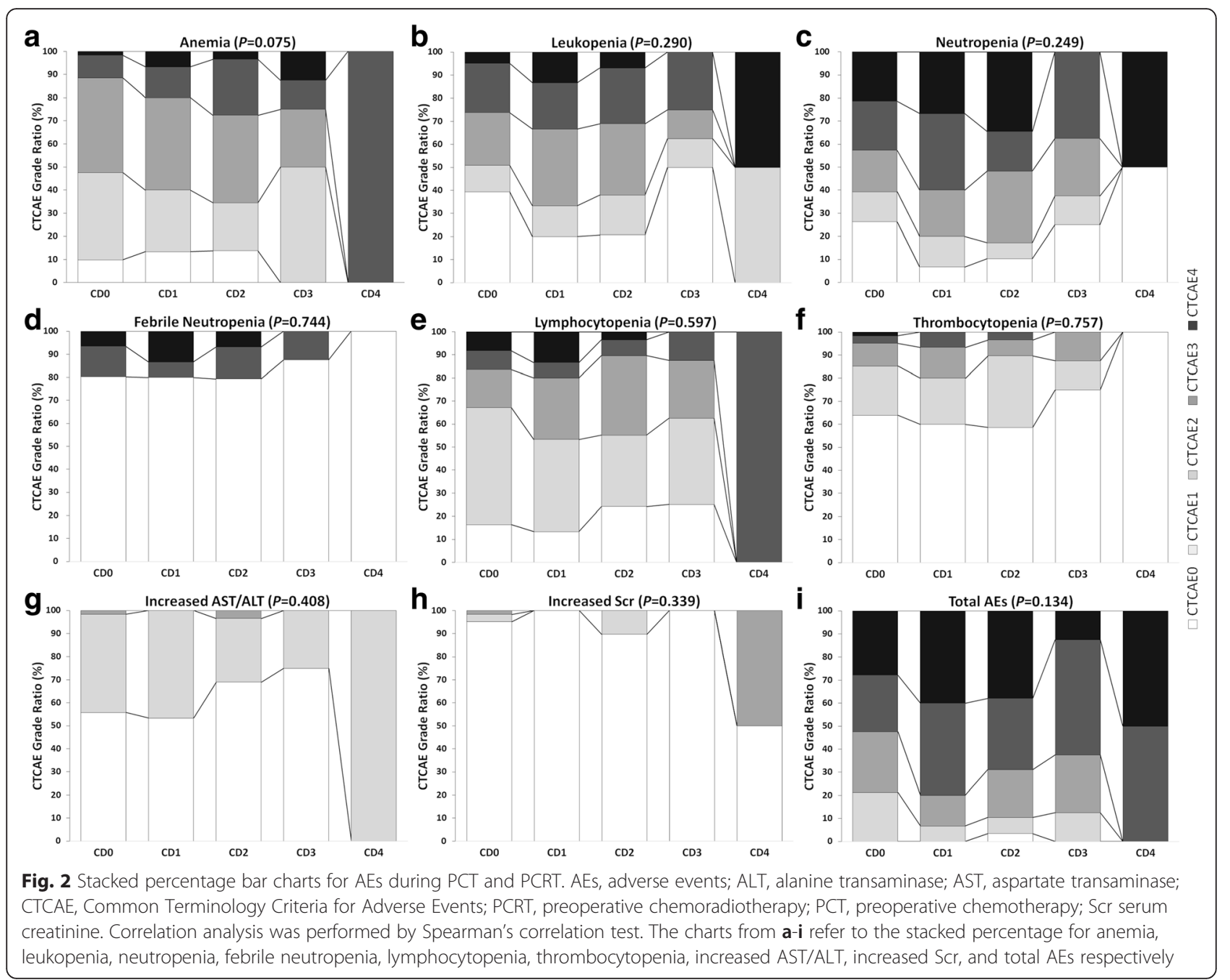

on postoperative complications after total gastrectomy plus D2 lymphadenectomy. However, because there were many discrepancies in patient selection, evaluation criteria, surgical procedures, and diagnostic standards among the previous studies, it was difficult to make definitive conclusions from these studies. The results of our current study can reduce concerns regarding the safety of surgery after PCT or PCRT, but they cannot eliminate them. A well-designed clinical trial is required to provide firm conclusions regarding this issue.

In previous studies, postoperative morbidity rates after gastrectomy plus lymphadenectomy varied from 25.7 to $45.7 \%$ in patients who underwent PCT or PCRT $[6,7,11,12]$. The morbidity rate of $47.0 \%$ in our study is relatively high but still comparable to the rate noted in a previous report from our institute [11]. In the present study, a high percentage of patients had stage IV cancer and underwent total gastrectomy and combined resection, which suggests that their surgery was more complicated. Postoperative morbidity may have been increased for this reason. It should be noted that the most common minor (CD1/2) complication in our study was pulmonary-related complications, a type of non-surgery-related complications. It is quite possible that these complications were related to a poor physical status of patients after PCT or PCRT. Additionally, the relatively low incidence of serious (CD3/4) complications $(8.7 \%)$ highlights the advantage of performing such complex surgery in a high-volume center.

Higher intraoperative blood loss and operative transfusion rates were previously reported to correlate with postoperative morbidity after gastrectomy and lymphadenectomy [11, 20]. In terms of $C D$ grades, we found significant differences among groups for EBL and transfusion rates. Patients with greater $\mathrm{CD}$ grades had a greater EBL. In the $\mathrm{CD}$ grading system, transfusion is classified as a grade 2 morbidity, which was why the transfusion rate was highest in our $\mathrm{CD} 1 / 2$ group. Generally, a higher blood loss reflects increased surgical difficulty and correlates with a higher transfusion rate. For the same level of surgical complexity, 


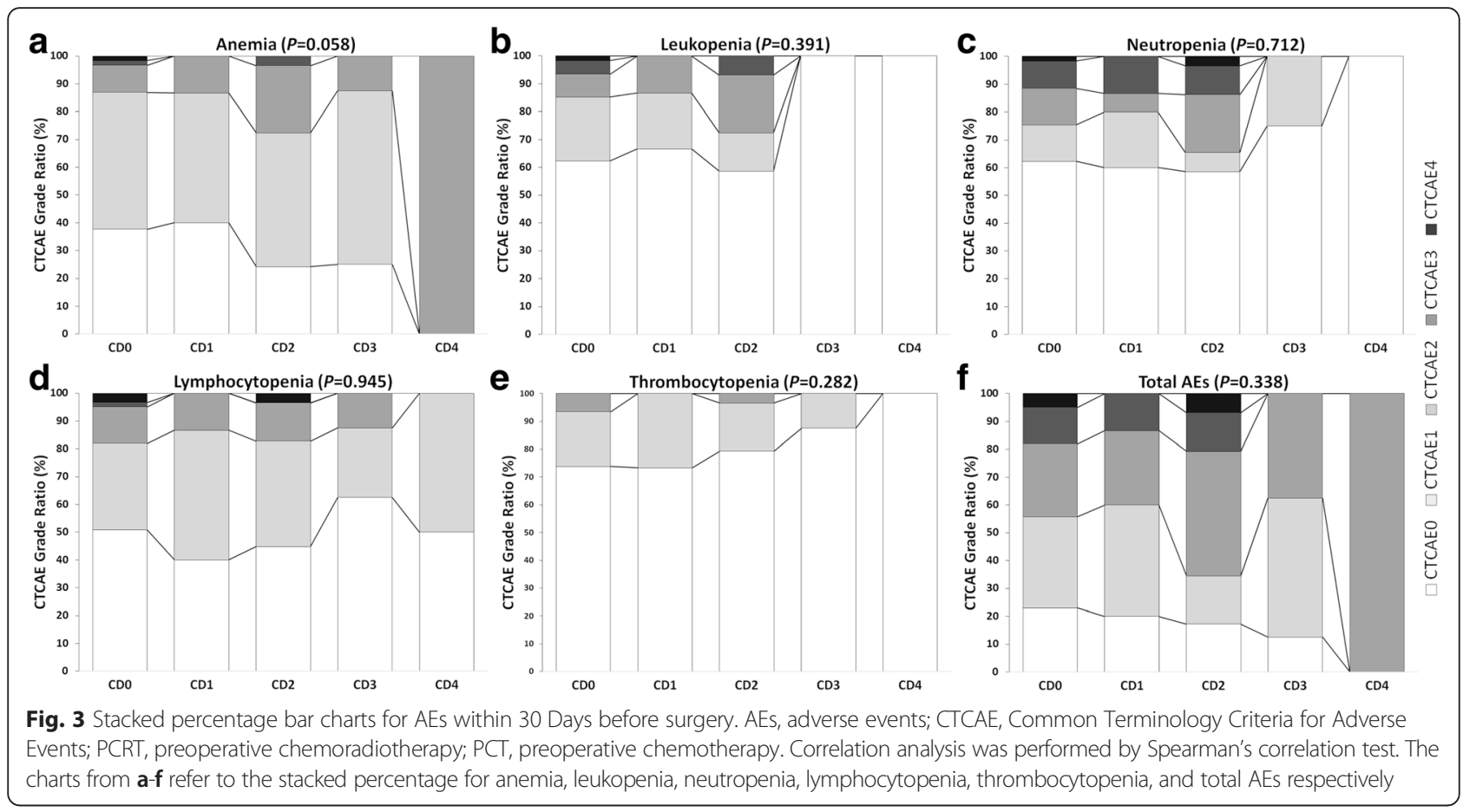

gastrectomy could be more difficult in patients who received PCT or PCRT because of peri-tumoral fibrosis, edema, or a bulky tumor mass. These factors are known to increase the need for surgical technique and experience. Independent of other factors related to postoperative morbidity, the surgical technique and quality are definitely crucial determinants of outcome. Meticulous manipulations during surgery should reduce trauma, operative blood loss, and ultimately morbidity.

In recent years, certain factors derived from routine laboratory investigations have been reported to manifest prognostic values in predicting the outcomes for patients with AGC and MGC. These factors include white blood cell and neutrophil counts, NLR, PLR, GPS, and PNI [14, 21, 22]. Importantly, Jiang et al. [23] reported that the severity of postoperative complication could influence the survival time for patients with gastric cancer, thereby suggesting the possibility of a direct link between the severity of postoperative morbidity and long-term prognostic factors. However, in our study, all laboratory findings, inflammation-based prognostic scores, and PNI did not differ among groups. The discrepancy is quite possibly because of the PCT or PCRT, as the baseline parameters no longer represented the patient's actual preoperative status, and the preoperative parameters could have been altered to varying degrees. Therefore, the above factors do not appear to be useful for predicting the postoperative morbidity for patients who received PCT or PCRT.

Limitations of this study include the relatively small number of patients and the natural drawbacks associated with all retrospective studies. There was substantial heterogeneity among patients and interventions. Firstly, patients with serious preoperative AEs tended to be under intensive clinical observations and may have received therapeutic interventions, which may have influenced postoperative morbidity. Secondly, the extent of surgery differed among patients. The surgical extent itself can influence postoperative morbidity, and should be well defined in a future study. Thirdly, the PCT and PCRT had different systemic and local effects, and each specific regimen also had unique toxicities. The variable treatments and regimens may have led to differing physiological alterations, even under the same $\mathrm{AE}$ grade. Fourthly, there were a number of patients who received preoperative treatment with PCT or PCRT but failed to subsequently proceed on to surgery. Data regarding the exact number of these patients nor the reasons why they did not subsequently proceed on to surgery was not included within the current study analysis. Therefore, had these individuals proceeded on to surgery after receiving preoperative treatment with PCT or PCRT, the observed degree and severity of postoperative complications that occurred in the overall study group could have been substantially different. However, there is no realistic way for us to make any such inferences into how preoperative AEs in this particular group of patients may have impacted upon the overall postoperative complications observed in the entire study group if this group of patients had theoretically proceeded on to surgery. Lastly, despite the limitations of our study, it is our belief that our use of both the CTCAE system for classifying AEs and the CD grading 
Table 6 Differences in laboratory tests, inflammation-based prognostic scores, and nutrition index according to Clavien-Dindo grades ${ }^{a}$

\begin{tabular}{|c|c|c|c|c|}
\hline & CD0 group $(n=61)$ & CD1/2 group $(n=44)$ & CD3/4 group $(n=10)$ & $P$ Value \\
\hline \multicolumn{5}{|l|}{ Baseline } \\
\hline $\mathrm{HB}(\mathrm{g} / \mathrm{L})$ & $129(66-155)$ & $121(64-170)$ & $112(72-159)$ & 0.284 \\
\hline WBC $\left(\times 10^{9} / L\right)$ & $5.9(3.6-13.9)$ & $6.4(3.6-14.2)$ & $8.1(3.9-10.2)$ & 0.109 \\
\hline $\operatorname{NEU}\left(\times 10^{9} / \mathrm{L}\right)$ & $3.6(2.2-10.9)$ & $3.9(2.0-11.7)$ & $4.8(1.4-7.7)$ & 0.136 \\
\hline $\operatorname{LYM}\left(\times 10^{9} / \mathrm{L}\right)$ & $1.7(0.7-3.1)$ & $1.8(0.6-3.0)$ & $2.0(1.4-2.4)$ & 0.238 \\
\hline $\operatorname{PLT}\left(\times 10^{9} / \mathrm{L}\right)$ & $298.0(96.0-639.0)$ & $295.0(141.0-599.0)$ & $301.5(166.0-438.0)$ & 0.784 \\
\hline$A L B(g / L)$ & $42.0(35.0-49.0)$ & $41.0(28.0-47.0)$ & $39.0(25.0-48.0)$ & 0.631 \\
\hline $\mathrm{ALT}(\mathrm{U} / \mathrm{L})$ & $14.0(5.0-75.0)$ & $14.5(5.0-58.0)$ & $13.0(7.0-59.0)$ & 0.888 \\
\hline AST (U/L) & $17.0(10.0-56.0)$ & $15.0(8.0-73.0)$ & $19.5(10.0-37.0)$ & 0.473 \\
\hline$A L P(U / L)$ & $56.0(24.0-109.0)$ & $59.5(25.0-123.0)$ & $71.5(31.0-99.0)$ & 0.137 \\
\hline $\mathrm{Scr}(\mathrm{mg} / \mathrm{dL})$ & $0.82(0.48-1.20)$ & $0.86(0.53-1.28)$ & $0.81(0.68-1.59)$ & 0.593 \\
\hline aPTT (second) & $29.8(23.0-37.2)$ & $29.6(24.9-38.6)$ & $28.6(24.9-33.1)$ & 0.315 \\
\hline PT (second) & $10.9(9.3-12.8)$ & $11.0(9.0-13.1)$ & $11.1(9.5-12.7)$ & 0.765 \\
\hline INR & $0.96(0.82-1.12)$ & $0.97(0.79-1.16)$ & $0.98(0.84-1.11)$ & 0.883 \\
\hline $\mathrm{PNI}$ & $50.2(40.4-61.8)$ & $50.6(34.2-60.0)$ & $49.7(33.0-60.2)$ & 0.954 \\
\hline NLR & $2.5(0.7-6.6)$ & $2.2(1.0-9.5)$ & $2.4(0.7-7.8)$ & 0.805 \\
\hline PLR & $181.9(67.6-557.7)$ & $179.1(73.8-487.0)$ & $171.1(78.3-256.9)$ & 0.683 \\
\hline \multicolumn{5}{|l|}{ Preoperative } \\
\hline $\mathrm{HB}(\mathrm{g} / \mathrm{L})$ & $116(86-152)$ & $115(76-160)$ & $117(91-125)$ & 0.810 \\
\hline WBC $\left(\times 10^{9} / \mathrm{L}\right)$ & $4.7(2.3-11.7)$ & $4.7(2.7-11.9)$ & $5.8(3.0-9.7)$ & 0.598 \\
\hline $\operatorname{NEU}\left(\times 10^{9} / \mathrm{L}\right)$ & $2.7(0.7-8.8)$ & $2.6(1.2-9.1)$ & $3.0(1.5-7.8)$ & 0.755 \\
\hline $\operatorname{LYM}\left(\times 10^{9} / \mathrm{L}\right)$ & $1.5(0.5-3.4)$ & $1.6(0.4-2.7)$ & $1.3(0.6-2.9)$ & 0.835 \\
\hline $\operatorname{PLT}\left(\times 10^{9} / \mathrm{L}\right)$ & $218.0(73.0-398.0)$ & $202.0(91.0-583.0)$ & $216.5(167.0-276.0)$ & 0.861 \\
\hline$A L B(g / L)$ & $40.0(27.0-48.0)$ & $39.5(26.0-52.0)$ & $39.0(32.0-41.0)$ & 0.541 \\
\hline $\mathrm{ALT}(\mathrm{U} / \mathrm{L})$ & $17.0(6.0-72.0)$ & $14.0(6.0-52.0)$ & $15.5(11.0-43.0)$ & 0.431 \\
\hline AST (U/L) & $20.0(12.0-75.0)$ & $19.0(11.0-42.0)$ & $19.0(12.0-42.0)$ & 0.671 \\
\hline$A L P(U / L)$ & $61.5(21.0-142.0)$ & $59.5(25.0-106.0)$ & $63.5(35.0-163.0)$ & 0.887 \\
\hline $\operatorname{Scr}(\mathrm{mg} / \mathrm{dL})$ & $0.78(0.43-1.39)$ & $0.78(0.38-1.55)$ & $0.75(0.63-1.34)$ & 0.874 \\
\hline $\mathrm{aPTT}$ (second) & $29.9(24.2-41.5)$ & $29.7(20.7-37.5)$ & $31.0(26.1-34.6)$ & 0.979 \\
\hline PT (second) & $10.5(9.4-13.1)$ & $10.9(9.4-13.4)$ & $10.5(9.6-11.7)$ & 0.196 \\
\hline INR & $0.93(0.83-1.15)$ & $0.97(0.83-1.18)$ & $0.94(0.85-1.01)$ & 0.147 \\
\hline $\mathrm{CRP}^{\mathrm{b}}(\mathrm{mg} / \mathrm{L})$ & $1.1(0.3-51.1)$ & $1.3(0.4-68.6)$ & $1.8(0.5-13.9)$ & 0.502 \\
\hline $\mathrm{PNI}$ & $46.8(30.5-59.9)$ & $46.0(33.2-62.1)$ & $45.4(37.2-54.3)$ & 0.801 \\
\hline NLR & $1.7(0.6-9.6)$ & $1.9(0.6-9.7)$ & $2.0(0.8-10.1)$ & 0.811 \\
\hline PLR & $134.6(38.2-574.1)$ & $123.4(44.7-640.7)$ & $148.3(66.1-392.1)$ & 0.714 \\
\hline $\mathrm{GPS}^{\mathrm{b}, \mathrm{c}}$ & & & & 0.804 \\
\hline Score 0 & $43(81.1)$ & $30(76.9)$ & $7(87.5)$ & \\
\hline Score 1 & $8(15.1)$ & $6(15.4)$ & $0(0.0)$ & \\
\hline Score 2 & $2(3.8)$ & $3(7.7)$ & $1(12.5)$ & \\
\hline $\mathrm{Pl}^{\mathrm{b}, \mathrm{c}}$ & & & & 0.572 \\
\hline Score 0 & $49(92.5)$ & $35(89.7)$ & $7(87.5)$ & \\
\hline Score 1 & $4(7.5)$ & $4(10.3)$ & $1(12.5)$ & \\
\hline
\end{tabular}

ALB, albumin; ALP, alkaline phosphatase; ALT, alanine transaminase; aPTT, activated partial thromboplastin time; AST, aspartate transaminase; CRP, C-reactive protein; GPS, Glasgow Prognostic Score; HB, hemoglobin; INR, international normalized ratio; LYM, lymphocyte count; NEU, neutrophil count; NLR, neutrophil-lymphocyte ratio; PI, Prognostic Index; PLR, platelet-lymphocyte ratio; PLT, platelet count; PNI, prognostic nutritional index; PT, prothrombin; Scr, serum creatinine; WBC, white blood cell count

${ }^{a}$ Data are presented as median (range) unless indicated otherwise

${ }^{b}$ Data retrieved from 100 patients, including 53 patients in CD0 group, 39 patients in CD1/2 group, and 8 patients in CD3/4 group

'Data are presented as number (percentage) 
system for classifying postoperative complications allowed for minimizing any subjectivity to the analysis process of the data included within the current study.

\section{Conclusions}

In summary, for patients with AGC and MGC who underwent gastrectomy and lymphadenectomy, this study provided supportive evidence favoring the use of PCT or PCRT. The CTCAE grades of preoperative AEs did not correlate with the $\mathrm{CD}$ grades of postoperative complications. The higher EBL and transfusion rate noted in patients with complications emphasize the importance of meticulous manipulations during surgery to reduce postoperative morbidity.

\section{Abbreviations}

AEs: Adverse events; AGC: Advanced gastric cancer; BMI: Body mass index; CD: Clavien-Dindo; CTCAE: Common terminology criteria for adverse events; EBL: Estimated blood loss; Gl: Gastrointestinal; GPS: Glasgow prognostic score; MGC: Metastatic gastric cancer; MIS: Minimal invasive surgery; NA: Not applicable; NLR: Neutrophil lymphocyte ratio; PCRT: Preoperative chemoradiotherapy; PCT: Preoperative chemotherapy; PI: Prognostic index; PLR: Platelet lymphocyte ratio; PNI: Prognostic nutritional index.

\section{Competing interests}

The authors declare that they have no competing interests.

\section{Authors' contributions}

SXL, SHS and YYC participated in study design, data collection and analysis. SXL and SHS wrote the manuscript. MN, JYA, HIK, JHC, WJH and SHN reviewed and revised the manuscript. All authors read and approved the final manuscript.

\section{Acknowledgements}

This study was supported by a grant from the National R\&D Program for Cancer Control, Ministry of Health and Welfare, Republic of Korea (1020390, 1320360). The authors thank Prof. Jiafu Ji for promoting the collaboration between Severance Hospital of Yonsei University and Peking University School of Oncology.

\section{Author details \\ ${ }^{1}$ Department of Surgery, Yonsei University Health System, Yonsei University College of Medicine, 50 Yonsei-ro, Seodaemun-gu, 120-752 Seoul, South Korea. ${ }^{2}$ Key laboratory of Carcinogenesis and Translational Research (Ministry of Education), Department of Gastrointestinal Surgery, Peking University Cancer Hospital \& Institute, Beijing, China. ${ }^{3}$ Department of Surgery, Inje University Busan Paik Hospital, Inje University College of Medicine, Busan, South Korea. ${ }^{4}$ Department of Gastric Surgery, Tokyo Medical and Dental University, Tokyo, Japan. ${ }^{5}$ Department of Surgery, Samsung Medical Center, Sungkyunkwan University School of Medicine, Seoul, South Korea.}

Received: 3 October 2015 Accepted: 11 January 2016

Published online: 19 January 2016

\section{References}

1. Ferlay J, Soerjomataram I, Dikshit R, Eser S, Mathers C, Rebelo M, et al. Cancer incidence and mortality worldwide: sources, methods and major patterns in GLOBOCAN 2012. Int J Cancer. 2015;136(5):E359-86.

2. Wu CW, Hsiung CA, Lo SS, Hsieh MC, Chen JH, Li AFY, et al. Nodal dissection for patients with gastric cancer: a randomised controlled trial. Lancet Oncol. 2006;7(4):309-15.

3. Songun I, Putter H, Kranenbarg EMK, Sasako M, van de Velde CJH. Surgical treatment of gastric cancer: 15-year follow-up results of the randomised nationwide Dutch D1D2 trial. Lancet Oncol. 2010;11(5):439-49.

4. Sasako M, Sakuramoto S, Katai H, Kinoshita T, Furukawa H, Yamaguchi T, et al. Five-year outcomes of a randomized phase III trial comparing adjuvant chemotherapy with S-1 versus surgery alone in stage II or III gastric cancer. J Clin Oncol. 2011;29(33):4387-93.
5. Noh SH, Park SR, Yang HK, Chung HC, Chung IJ, Kim SW, et al. Adjuvant capecitabine plus oxaliplatin for gastric cancer after D2 gastrectomy (CLASSIC): 5-year follow-up of an open-label, randomised phase 3 trial. Lancet Oncol. 2014;15(12):1389-96.

6. Cunningham D, Allum WH, Stenning SP, Thompson JN, Van de Velde CJ, Nicolson $\mathrm{M}$, et al. Perioperative chemotherapy versus surgery alone for resectable gastroesophageal cancer. N Engl J Med. 2006;355(1):11-20.

7. Ychou M, Boige V, Pignon JP, Conroy T, Bouche O, Lebreton G, et al. Perioperative chemotherapy compared with surgery alone for resectable gastroesophageal adenocarcinoma: an FNCLCC and FFCD multicenter phase III trial. J Clin Oncol. 2011;29(13):1715-21.

8. Macdonald JS, Smalley SR, Benedetti J, Hundahl SA, Estes NC, Stemmermann GN, et al. Chemoradiotherapy after surgery compared with surgery alone for adenocarcinoma of the stomach or gastroesophageal junction. N Engl J Med. 2001;345(10):725-30.

9. Schildberg CW, Croner R, Merkel S, Schellerer V, Muller V, Yedibela S, et al. Outcome of operative therapy of hepatic metastatic stomach carcinoma: a retrospective analysis. World J Surg. 2012;36(4):872-8.

10. Saito M, Kiyozaki H, Takata O, Suzuki K, Rikiyama T. Treatment of stage IV gastric cancer with induction chemotherapy using S-1 and cisplatin followed by curative resection in selected patients. World J Surg Oncol. 2014;12:406.

11. An JY, Kim KM, Kim YM, Cheong JH, Hyung WJ, Noh SH. Surgical complications in gastric cancer patients preoperatively treated with chemotherapy: their risk factors and clinical relevance. Ann Surg Oncol. 2012:19(8):2452-8

12. Fujitani K, Ajani JA, Crane CH, Feig BW, Pisters PW, Janjan N, et al. Impact of induction chemotherapy and preoperative chemoradiotherapy on operative morbidity and mortality in patients with locoregional adenocarcinoma of the stomach or gastroesophageal junction. Ann Surg Oncol. 2007;14(7): 2010-7.

13. Japanese Gastric Cancer A. Japanese gastric cancer treatment guidelines 2010 (ver. 3). Gastric Cancer. 2011;14(2):113-23. doi:10.1007/s10120-011-0042-4.

14. Li QQ, Lu ZH, Yang L, Lu M, Zhang XT, Li J, et al. Neutrophil count and the inflammation-based glasgow prognostic score predict survival in patients with advanced gastric cancer receiving first-line chemotherapy. Asian Pac J Cancer Prev. 2014:15(2):945-50.

15. National Cancer Institute. Common Toxicity Criteria (CTC) Version 4.0.3. http://evs.nci.nih.gov/ftp1/CTCAE/CTCAE_4.03_2010-06-14_QuickReference_ 8.5x11.pdf. Accessed August 6, 2010.

16. Dindo D, Demartines N, Clavien PA. Classification of surgical complications: a new proposal with evaluation in a cohort of 6336 patients and results of a survey. Ann Surg. 2004;240(2):205-13.

17. Tsuburaya A, Mizusawa J, Tanaka Y, Fukushima N, Nashimoto A, Sasako M, et al. Neoadjuvant chemotherapy with S-1 and cisplatin followed by D2 gastrectomy with para-aortic lymph node dissection for gastric cancer with extensive lymph node metastasis. Br J Surg. 2014;101(6):653-60.

18. Jung DH, Lee HJ, Han DS, Suh YS, Kong SH, Lee KU, et al. Impact of perioperative hemoglobin levels on postoperative outcomes in gastric cancer surgery. Gastric Cancer. 2013;16(3):377-82.

19. Reim D, Huser N, Humberg D, Novotny A, Assfalg V, Matevossian E, et al. Preoperative clinically inapparent leucopenia in patients undergoing neoadjuvant chemotherapy for locally advanced gastric cancer is not a risk factor for surgical or general postoperative complications. J Surg Oncol. 2010:102(4):321-4

20. Ishino $Y$, Saigusa S, Ohi M, Yasuda H, Tanaka K, Toiyama Y, et al. Preoperative $\mathrm{C}$-reactive protein and operative blood loss predict poor prognosis in patients with gastric cancer after laparoscopy-assisted gastrectomy. Asian J Endosc Surg. 2014;7(4):287-94.

21. Migita K, Takayama T, Saeki K, Matsumoto S, Wakatsuki K, Enomoto K, et al. The prognostic nutritional index predicts long-term outcomes of gastric cancer patients independent of tumor stage. Ann Surg Oncol. 2013;20(8):2647-54

22. Jiang N, Deng JY, Liu Y, Ke B, Liu HG, Liang H. The role of preoperative neutrophil-lymphocyte and platelet-lymphocyte ratio in patients after radical resection for gastric cancer. Biomarkers. 2014;19(6):444-51.

23. Jiang N, Deng JY, Ding XW, Zhang L, Liu HG, Liang YX, et al. Effect of complication grade on survival following curative gastrectomy for carcinoma. World J Gastroenterol. 2014;20(25):8244-52. 